\title{
Leveraging virtual reality for student development of force models in the introductory lab
}

Jared P. Canright, Jack R. Olsen, and Suzanne White Brahmia

Department of Physics, University of Washington, 3910 15th Ave NE, Seattle, WA, 98195

Providing students with opportunities to develop models in the introductory physics lab is made difficult by the dearth of physical phenomena that are both accessible to students at the introductory level and aren't easily found with a Google search. In response to this difficulty, we have developed a set of laboratory activities that leverage the unique affordances of virtual reality to provide a learning opportunity in which introductory physics students engage in the authentic practice of physics model development for a novel phenomenon. We present preliminary evidence of expert-like modeling behaviors, both in the context of hypothesis generation and in the context of modeling a measurement tool, as well as suggestions how virtual reality labs can be engineered to target other laboratory learning outcomes. Further, we discuss difficulties associated with this approach to curriculum design and ways they might be mitigated.

\footnotetext{
2020 PERC Proceedings edited by Wolf, Bennett, and Frank; Peer-reviewed, doi.org/10.1119/perc.2020.pr.Canright Published by the American Association of Physics Teachers under a Creative Commons Attribution 4.0 license.
} Further distribution must maintain the cover page and attribution to the article's authors. 


\section{INTRODUCTION}

Modern virtual reality (VR) technology offers unique affordances, leveraging stereoscopic 3D rendering and embodied input modalities to create immersive learning environments that offer new ways to enrich student learning in physics [1]. Despite the possibilities VR enables, or perhaps in part due to their sheer variety, quantitative evidence of VR improving physics learning outcomes remains elusive. Prior research has compared the effectiveness of a VR intervention with the effectiveness of equivalent desktop computerbased and/or hands-on interventions at teaching introductory physics content. Application of this methodology to Moon phases [2], electrostatics [3], and magnetostatics [4] has found that students generally prefer VR treatments, but their enthusiasm is not accompanied by measurable learning gains over other treatments.

We describe here a pilot study of a different paradigm of VR use in the physics classroom. Rather than applying VR as another medium through which to teach existing physics content, we seek to employ it as a means to engineer physical phenomena and the tools available to examine those phenomena. In doing so, we can target specific physics laboratory learning outcomes in an instructional context where those outcomes are otherwise difficult to achieve.

We specifically seek to enable the authentic practice of physics model generation in an introductory electromagnetism laboratory. This goal is motivated by difficulties moving away from "verification" labs in high-enrollment introductory courses, which have been characterized as "exercises that verify known answers to unexciting questions" [5] and criticized for failure to measurably improve students' understanding of physics content [6] or foster expert-like views of experimental physics [7]. Physics model generation is recognized as a key learning goal of undergraduate physics laboratories by the AAPT [8] and is the cornerstone of the Investigative Science Learning Environment (ISLE) [9], a researchvalidated instructional system that develops expert-like beliefs and habits of mind about experimental physics.

The dearth of physical phenomena that are accessible at the introductory level for which students cannot readily find the "right" answer presents challenges for development of inquiry labs. Introductory students often consider any known model to be a standard by which their data is measured, rather than a conjecture subject to refinement by experimentation. Stein, Smith, and Holmes found that even in an inquiryfocused lab curriculum, at least $30 \%$ of student groups engaged in questionable research practices in attempts to make their data (incorrectly) confirm the model under investigation [10]. Hu, Zwickl, Wilcox, and Lewandowski conducted a qualitative study into students' belief that physics experiments can be completed without an understanding of the involved physical concepts and mathematical relationships. The authors concluded that, “... incorporating some lab activities for which the outcome is not known... might have a significant impact on students' understanding of the impor- tance of experimental physics as a mechanism for uncovering new physics and driving the creation of new theoretical models." [11]

The Novel Observations in Mixed Reality (NOMR) lab project addresses this challenge by presenting representations of familiar and fictitious physical phenomena in an interactive 3D virtual learning environment (VLE) experienced via VR. Introduction of novel physical phenomena known only to the developer completely eliminates students' ability to identify an existing model or other "source of truth." Their sole source of information about novel phenomena is their own observations. A model-generating NOMR lab activity was pilot-tested with students enrolled in an introductory electromagnetism course. This paper seeks to document:

- The design of the NOMR labs, the phenomena explored within them, and the tools available to explore those phenomena;

- Preliminary evidence of expert-like modeling behavior employed in students' work in a NOMR lab;

- Discussion of opportunities to employ this approach to target other laboratory learning outcomes;

- Difficulties associated with this approach and strategies for their mitigation.

\section{CONTEXT AND METHODS}

\section{A. Instructional Context}

This Winter 2020 study took place in the honors introductory electromagnetism course at the University of Washington (UW), the second term of a three-quarter course where ISLEbased labs are being developed as part of the UW Laboratory Transformation Project. The lab sections met for two hours weekly. The course enrollment of 26 collaborated as eight persistent groups, four groups in each of two lab sections.

The 1-week NOMR lab activity that is the focus of this paper was the final lab of the quarter. Earlier in the term, students participated in a 2-week NOMR lab. Students quantitatively tested a model for interactions between VR analogues of electrically charged particles, and then developed and tested a model (Coulomb's law) for interactions between unfamiliar, fictitious "minty" particles. Minty particles carry a single charge type which repels itself at close range and attracts at long range, obeying a force law that resembles Coulomb's law with an additional constant negative term. Students developed strategies for using virtual measuring tools to gather force and separation data to develop a model, and became adept at using the VR technology. In the rest of the paper, we report on the later NOMR lab, in which students built on these skills. 


\section{B. Virtual Learning Environment}

We refer to the software package running on the VR system in which students carry out the lab activity as the virtual learning environment (VLE). We developed the VLEs described below in the Unity game engine [12]. The VLE provided the particles for consideration, distance and force measurement tools (shown in Figure 1), and other capabilities:

Force meter: Force meters analogous to familiar spring scales consist of a pivot anchored at a point in space, a bob to which a single particle can be attached, and a rigid rod of fixed length connecting the pivot and bob. When a particle is attached to the bob, it swings around the pivot in response to forces upon the particle, coming to rest where the unit vector $\hat{r}$ pointing from the pivot to the bob is parallel to the force experienced by the particle $\overrightarrow{\mathbf{F}}$. The meter displays a scalar value equal to the dot product of these quantities: $F=\overrightarrow{\mathbf{F}} \cdot \hat{r}$, expressed in arbitrary force units. This can be thought of as the scalar force felt by a rigid spring connecting the particle and the pivot, with positive and negative values representing extension and compression of the spring, respectively.

Measuring tape: Two small spheres connected by a line accompany a display that gives the distance in meters between the spheres.

Anchors: Particles can be anchored, fixing their position at a point in space and preventing them from moving in response to collisions or field-mediated interactions.

Toggling particle interactions: A "Toggle Physics" button allows the user to freeze/unfreeze all particles in the scene, allowing for configuration of experiments.

Paint: Particles all start out green regardless of their identity, and can be colored yellow, purple, black, or white as a visual aid.

We refer to a collection of distinct particles in the VR lab and the relationships which govern their interactions as a scenario. All particles' charge type (if applicable) and magnitude are immutable, and all have the same immutable mass. Each particle produces a field $\overrightarrow{\mathbf{E}}_{\text {type }}$ associated with its charge type $q_{\text {type }}$. Each field independently obeys the superposition principle.

Each of the four groups within a lab section explored a different scenario; we report on two scenarios in sections III and IV. Students were informed that each scenario had no more than three distinct particles.

\section{Laboratory Setup and Directions}

Each group of two to four students was provided two identical setups, each consisting of a desktop computer and an Oculus Quest [13] VR system. While a student operated the VLE, the VR headset display was mirrored to the desktop computer via scrcpy [14] such that their partner(s) could see what was happening and record observations. Students were prompted to take turns using the headset.

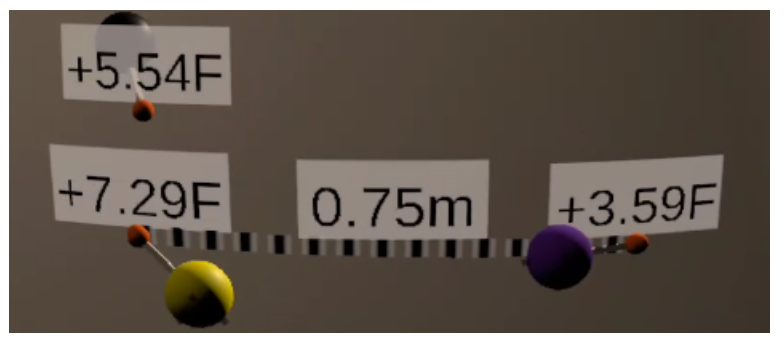

FIG. 1. This screenshot from the Triforce scenario shows power (yellow), wisdom (black), and courage (purple) particles interacting, each attached to a force meter. A measuring tape displays the distance between the pivots of the force meters attached to the power and courage particles.

Each student received the same lab procedure handout at the start of the lab; none were previously briefed on the lab content. Students were first directed to develop observational experiments to answer the questions:

- How many types of particles does the VR lab generate?

- How can the identity of a particle be determined?

Once students identified the number of particle types present in their scenario, named them, and developed a procedure for identifying them, they were directed to identify the independent variable(s) relevant to their particles' interactions and develop an evidence-backed mathematical model relating one such variable to the force between particles.

\section{Research Methodology}

While this paper is meant largely as a presentation of a unique curriculum design, case studies of two student groups' activities during the lab are presented in the following sections. Video data of select groups' lab activities were recorded and transcribed, and enhanced by field notes taken by the first author. These data were used to reconstruct a picture of notable moments in students' work during the lab activity.

\section{TAGALONG SCENARIO}

This scenario's only particle (the tagalong) produces a field dependent upon the relative velocity of the source particle $\overrightarrow{\mathbf{v}}_{\text {src }}$ and the velocity of the inertial reference frame $\overrightarrow{\mathbf{v}}_{\text {frame }}$ in which the field is measured, using Galilean relativity:

$$
\overrightarrow{\mathbf{E}}_{\text {tag }}\left(\overrightarrow{\mathbf{v}}_{\text {src }}, \overrightarrow{\mathbf{v}}_{\text {frame }}\right)=q_{\text {tag }, s r c}\left(\overrightarrow{\mathbf{v}}_{\text {src }}-\overrightarrow{\mathbf{v}}_{\text {frame }}\right) .
$$

A tagalong with charge $q_{t a g, i}$ experiencing a net tagalong field $\overrightarrow{\mathbf{E}}_{t a g}$ as measured in its rest frame experiences a force proportional to the field:

$$
\overrightarrow{\mathbf{F}}_{t a g, i}=q_{t a g, i} \overrightarrow{\mathbf{E}}_{t a g} .
$$




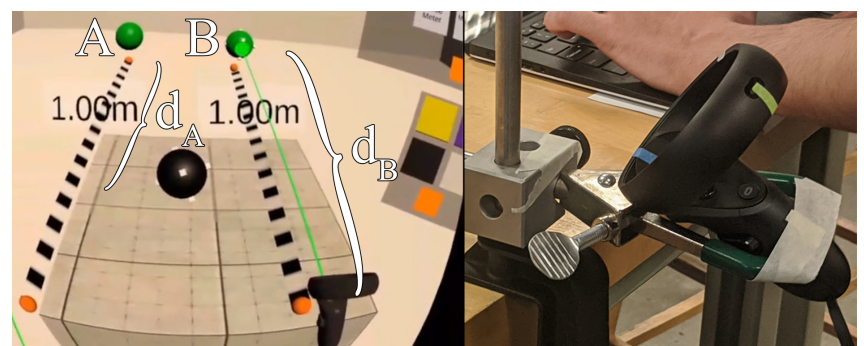

FIG. 2. Left: The Scouts' experimental setup in the VLE, with $N=1$ black anchored tagalong between free tagalong A and manipulated tagalong B. Right: The Oculus Quest controller taped to the lab stand held $\mathrm{B}$ on a fixed path. With this configuration, moving B a distance $d_{B}=1 \mathrm{~m}$ toward the controller at a constant velocity causes A to move $d_{A} \approx 0.5 \mathrm{~m}$ in the same direction before coming to rest.

This force causes a system of tagalongs to tend toward a state where its linear momentum is distributed equally among all tagalongs. Thus, if one tagalong in a moving system is anchored, the linear momentum of the system quickly goes to zero as every other tagalong slows to a halt.

A pair of students whom we will refer to as the Scouts worked through the Tagalong scenario. Through exploration of the behavior of a system of many tagalongs in response to manipulation of a single tagalong, the Scouts developed an initial model: "Particles accelerate to match the average velocity of particles around it." Casting this verbal representation into mathematical form results in the relationship implemented to govern this scenario: $\overrightarrow{\mathbf{F}}_{\text {tag }, i} \propto \overrightarrow{\mathbf{v}}_{\text {avg }}$. In the process of developing a quantitative experiment, the Scouts determined that every particle behaved the same way, and that their interactions were independent of relative position.

To test this model, they proposed to anchor $N$ tagalongs, leave one tagalong (A) floating freely, and move another tagalong (B) a specific distance $d_{B}$ at a constant velocity $\vec{v}_{B}$. Thus, they reasoned, the velocity to which the free particle would try to accelerate is given by $v_{a v g}=v_{B} / N$ for the time particle $\mathrm{B}$ is in motion $t=d_{B} / v_{B}$, after which $v_{B}$ and $v_{a v g}$ return to zero. The Scouts' model predicted that the distance traveled by the free particle $d_{A}$ would be proportional to $v_{a v g}$, and thus inversely proportional to $N: d_{A} \propto 1 / N$. The Scouts set out to measure $d_{A}$ for various $N$ to test this hypothesis.

When setting up this experiment, the Scouts recognized that they needed a means by which to keep $d_{B}$ and $\vec{v}_{B}$ constant between trials. The VR controllers can move a particle away from or toward the controller in a straight line by moving the controller thumbstick. The Scouts suspected (and confirmed with a TA) that pushing the thumbstick all the way up or all the way down caused the held particle to move at a constant velocity $v_{B}$. The Scouts found a very creative way to move $\mathrm{B}$ along the same path every trial, keeping consistent $d_{B}$ and $\hat{\mathbf{v}}_{B}$ : They taped a controller to a lab stand as in Figure 2 to fix its position for the entire experiment, never letting go of B.
The experimental design and configuration of apparatus performed by the Scouts demonstrates NOMR labs' potential to create an opportunity for students to exercise incredible creativity in experiment design in an introductory lab. However, their accurate model was backed by a flawed analysis. Their assumption that $v_{a v g}=v_{B} / N$ should have been $v_{\text {avg }}=v_{B} /(N+1)$ to account for the influence of particle B. While the Scouts recorded $d_{A}$ for $N=0$ and $N=1$, these data were absent in the Scouts' plot of $1 / N$ versus $d_{A}$, which they presented to support their ultimate conclusion that $d_{A} \propto 1 / N$.

\section{TRIFORCE SCENARIO}

The Triforce scenario features three unique particles, each with its own charge type and associated field; these are dubbed power, wisdom, and courage. For brevity, we describe their interactions as a set of force laws governing interactions between pairs of particles, all of which satisfy Newton's third law. The force exerted on a particle of type 2 by a particle of type 1 is given by

$$
\overrightarrow{\mathbf{F}}_{1 \leftrightarrow 2}=k_{1,2} C \frac{\hat{r}}{r^{2}},
$$

where $C$ is a constant of proportionality independent of particle type and $k_{1,2}=+1,-1$, or 0 for attractive, repulsive, or zero force, respectively, as given for each combination of particle types in Table I.

TABLE I. Interactions between pairs of Triforce particles.

\begin{tabular}{c|c|c|c}
\hline \hline 1 & Power & Wisdom & Courage \\
\hline Power & Attraction & Repulsion & Attraction \\
\hline Wisdom & Repulsion & Attraction & Zero \\
\hline Courage & Attraction & Zero & Repulsion \\
\hline \hline
\end{tabular}

A group of three students to whom we will refer as the Trio worked through the Triforce scenario. The process of determining the number and properties of the particles present in this scenario was more involved due to the presence of three unique particles. This process was confounded by a software bug that teleported a courage particle behind a wall in the VLE, where it remained, attracting other power and courage particles. The rogue courage particle was only discovered and deleted after a TA was asked why particles were being mysteriously attracted to a wall:

TA: "I mean, maybe the force is asymmetric, but..."

Student 1: "...we could reset and try this again."

Student 2, in headset: "I don't like the idea of a force being asymmetric. That, like, breaks the second law."

Student 3: "Wait, the force shouldn't be asymmetric, should it?"

Student 2: "Well, it shouldn't, but this is a simulation, so..." 
Student 1: "Should we try resetting, just to make sure we don't have any stray particles?"

Student 2: Agrees and resets the VLE.

This exchange shows that students briefly entertained the notion that the particles' strange tendency to be attracted in a certain direction was a result of deliberate violation of Newton's laws in the VLE. While this is a promising example of students' willingness to consider a variety of explanations for a phenomenon, it also demonstrates that VLE bugs are dangerous. Had the Trio not reset the VLE, this bug may have led them down a frustrating rabbit hole.

With the bug resolved, the Trio soon arrived at the conclusion that their scenario had three unique particles and successfully identified the nature of the interaction between each pair of particles, constructing Table I. At this point, we observed that by giving a three-student group a scenario with three unique particles, we laid the groundwork for an unanticipated expert-like behavior: The students named the particles after themselves.

In their presentation, the Trio presented quantitative evidence for a $\overrightarrow{\mathbf{F}}_{p \leftrightarrow p} \propto \frac{\hat{r}}{r^{2}}$ attractive force law between two power particles, and suggested that other pairs obey the same law but noted that this claim was untested.

\section{DISCUSSION}

This work demonstrated a pilot physics laboratory curriculum design which leverages the unique affordances of VR to enable authentic physics model development at the introductory level. The groups who worked with the Tagalong and Triforce scenarios both demonstrated expert-like modeling behavior, identified using the ISLE scientific ability rubrics for observational experimentation [15]. Both clearly identified and described the salient features and patterns of their scenario in words and connected that representation to a databacked mathematical relationship that accurately characterized parts of their scenario.

In light of successful observational experimentation, the Scouts' trip-up in their testing experiment raises interesting questions. They found that measurements of $d_{A}$ for $N=2 \ldots 7$ fit a believably linear trend in a plot of $d_{A}$ versus $1 / N$, whereas $N=0,1$ did not. To resolve this discrepancy, the Scouts omitted $N=0,1$ in that plot. It is unclear why the Scouts found fault in those data, rather than their analysis or their model. Video data suggest that the Scouts (rightly) believed their model $\overrightarrow{\mathbf{F}}_{t a g} \propto \overrightarrow{\mathbf{v}}_{\text {avg }}$ was well-supported by qualitative observational experiments. This suggests that the Scouts were unable or did not attempt to resolve the discrepancy by examining the relationship between their model and their derived experimental prediction $d_{A} \propto 1 / N$. Further study of students' use of questionable scientific practices in absence of a "correct" model may aid development of inquiry-based lab curriculum.

This trial suggested that NOMR labs may enable other laboratory learning goals:
Modeling measurement tools: We were concerned that students may misinterpret the force meter's signed scalar readout of a vector quantity, but it turned out to be a robust tool with just enough depth to require careful thought but not obstruct students' ability to conduct experiments. Examination of student modeling of virtual measurement tools through a framework which treats that process as a partner to modeling a physical system [16] may inform the design of NOMR labs which intentionally build this skill.

Designing experiments: Given the same set of measurement tools, the Tagalong scenario required a much more complicated experimental design than Triforce. By careful consideration of what tools are available in a NOMR lab and how they must be combined to make sense of a phenomenon, one could regulate the complexity of experiments expected of students.

We also find that a VR lab is not without its difficulties:

Implementation and facilitation of $V R$ in the classroom comes with significant monetary and technical overhead, and requires special TA training and protocols to ensure safety of students using the headsets and those around them.

$V L E$ creation currently requires significant VR development experience, which is generally not expertise readily available in a physics department. By exploring this approach further, the necessary elements of physics VLE authoring tools may be identified, paving the way for development of authoring tools designed to be used by physics educators.

VLE physics bugs are an epistemological hazard. As found in Section IV, unintended physics bugs can be interpreted as intentional behavior by students. If their VLE had included the ability to create uniform global fields or charge walls, the bug may have gone unnoticed for the duration of the lab. Comparing how students think about troubleshooting VR tools and physical tools is a promising area for further study.

These findings beg the question: What unique advantage does VR lend to the NOMR labs over desktop simulations? We have a sense that VR produced effects that could not be replicated on 2D screens, but this study does not aim to make that argument. Our experience is consistent with Greenwald's findings that direct connection of sensorimotor inputs with dynamic simulations, blending of multiple representations, and reduced cognitive load are all likely contributors to any unique benefit associated with VR physics curriculum [1]; future work will draw from his methodology to provide a more concrete answer to this question.

\section{ACKNOWLEDGMENTS}

The authors thank Robert Pecoraro and Charlotte Zimmerman for their invaluable effort, and gratefully acknowledge financial support from the National Science Foundation GRF (DGE-1256082), ARCS Foundation, AAPT Bauder Fund, and UW Student Technology Fee. 
[1] S. W. Greenwald, The Equipped Explorer: Virtual Reality as a Medium for Learning, Ph.D. thesis, MIT (2018).

[2] J. H. Madden, A. S. Won, J. P. Schuldt, B. Kim, S. Pandita, Y. Sun, T. J. Stone, and N. G. Holmes, Virtual Reality as a Teaching Tool for Moon Phases and Beyond, in PERC Proceedings (Washington, DC, 2018).

[3] J. R. Smith, A. Byrum, T. M. McCormick, N. Young, C. Orban, and C. D. Porter, A controlled study of stereoscopic virtual reality in freshman electrostatics, in PERC Proceedings (Cincinnati, OH, 2017) pp. 376-379.

[4] C. D. Porter, J. R. Brown, J. R. Smith, E. M. Stagar, A. Simmons, M. Nieberding, A. Ayers, and C. Orban, A controlled study of virtual reality in first-year magnetostatics, in Physics Education Research Conference 2019, PER Conference (Provo, UT, 2019).

[5] R. Trumper, The Physics Laboratory - A Historical Overview and Future Perspectives, Science and Education 12, 645 (2003).

[6] N. G. Holmes, J. Olsen, J. L. Thomas, and C. E. Wieman, Value added or misattributed? A multi-institution study on the educational benefit of labs for reinforcing physics content, Physical Review Physics Education Research 13, 10.1103/PhysRevPhysEducRes.13.010129 (2017).

[7] B. R. Wilcox and H. J. Lewandowski, Developing skills versus reinforcing concepts in physics labs: Insight from a survey of students' beliefs about experimental physics, Physical Review Physics Education Research 13, 10.1103/PhysRevPhysEducRes.13.010108 (2017).

[8] J. Kozminski, H. Lewandowski, N. Beverly, S. Lindaas,
D. Deardorff, A. Reagan, R. Dietz, R. Tagg, M. Eblen-ÂZayas, J. Williams, R. Hobbs, and B. Zwickl, AAPT Recommendations for the Undergraduate Physics Laboratory Curriculum, Tech. Rep. (AAPT, 2014).

[9] E. Etkina and A. V. Heuvelen, Investigative Science Learning Environment - A Science Process Approach to Learning Physics, in Research-Based Reform of University Physics, Vol. 1 (2007).

[10] M. M. Stein, E. M. Smith, and N. G. Holmes, Confirming what we know: Understanding questionable research practices in intro physics labs, in PERC Proceedings (Washington, DC, 2018).

[11] D. Hu, B. M. Zwickl, B. R. Wilcox, and H. J. Lewandowski, Qualitative investigation of students' views about experimental physics, Physical Review Physics Education Research 13, 10.1103/PhysRevPhysEducRes.13.020134 (2017).

[12] Unity Technologies, Unity Real-Time Development Platform.

[13] Oculus Quest.

[14] scrcpy Github page.

[15] E. Etkina, A. Van Heuvelen, S. White-Brahmia, D. T. Brookes, M. Gentile, S. Murthy, D. Rosengrant, and A. Warren, Scientific abilities and their assessment, Physical Review Special Topics - Physics Education Research 2, 10.1103/PhysRevSTPER.2.020103 (2006).

[16] B. M. Zwickl, D. Hu, N. Finkelstein, and H. J. Lewandowski, Model-based reasoning in the physics laboratory: Framework and initial results, Physical Review Special Topics - Physics Education Research 11, 10.1103/PhysRevSTPER.11.020113 (2015). 